\title{
ON BOUNDED SETS IN INDUCTIVE LIMITS OF NORMED SPACES
}

\author{
KLAUS FLORET
}

\begin{abstract}
A theorem on bounded sets in locally convex inductive limits is proven and applied in various special cases.
\end{abstract}

1. For many questions in analysis in locally convex spaces it is of crucial importance to know how the bounded sets behave. In particular in locally convex inductive limits of (injective) sequences of normed spaces $E=$ ind $_{n \rightarrow} E_{n}$ there are various examples given by B. M. Makarov [12] with the property that bounded sets in $E$ are not situated in any $E_{n}$, or are not bounded there. It is the purpose of this note to give a sufficient condition for a sequence $\left(E_{n}\right)$ of normed spaces to generate regularly the inductive limit $E=$ ind $_{n \rightarrow} E_{n}$, i.e., every bounded set in $E$ is situated in some $E_{n_{0}}$ and bounded there. Since the closure of $\{0\}$ in $E$ is bounded in $E$, regular inductive limits are Hausdorff; this observation yields an easy proof and a slight improvement (see \$7) of a frequently used result of E. Dubinsky [3] for an inductive limit to be Hausdorff.

2. The terminology is that of G. Köthe [10] and [6]. The inductive sequences

$$
E_{1} \stackrel{\pi_{1}}{\rightarrow} E_{2} \stackrel{\pi_{2}}{\rightarrow} E_{3} \stackrel{\pi_{3}}{\rightarrow} \ldots
$$

consist of separated locally convex spaces $E_{n}$ and injective (continuous) linking mappings $\pi_{n}: E_{n} \rightarrow E_{n+1}$; this means that the generating spaces can be considered as linear subspaces of $E=$ ind $_{n \rightarrow} E_{n}$, the linking mappings now being simply the inclusion mappings. Denoting by $\tau_{n}$ the topology of $E_{n}$ and by $\tau$ the (locally convex) inductive limit topology of $E=\cup_{n=1}^{\infty} E_{n}$, the sequence $\left(E_{n}\right)$ is called:

(a) $\alpha$-regular, if every $\tau$-bounded subset of $E$ is situated in some $E_{n}$,

(b) $\beta$-regular, if every $\tau$-bounded subset of $E$, which is situated in some $E_{n}$, is $\tau_{m}$-bounded for some $m \in \mathbf{N}$,

(c) regular, if it is both $\alpha$ - and $\beta$-regular, i.e. for every $\tau$-bounded subset $A \subset E$ there is an $n \in \mathbf{N}$ such that $A \subset E$ and $A$ is $\tau_{n}$-bounded.

The mentioned counterexamples of B. M. Makarov are $(L B)$-spaces which show that (a) $\nrightarrow(\mathrm{b}),(\mathrm{b}) \nrightarrow(\mathrm{a})$ and that an $(L B)$-space can be neither $\alpha$ - nor

Received by the editors February 22, 1978.

AMS (MOS) subject classifications (1970). Primary 46A05, 46A45, 46B10.

Key words and phrases. Locally convex inductive limits, bounded sets, sequence space, reflexive Banach-space. 
$\beta$-regular (taking for example the direct sum of the former counterexamples).

It is obvious that the regularity properties of a sequence are inherited by equivalent sequences. The inductive limit of a regular sequence of different metrizable spaces is never metrizable [5, p. 94].

3. Grothendieck's factorization theorem for $(L F)$-spaces [7, I, p. 16] implies that a Hausdorff $(L F)$-space is regular if and only if it is Mackey-complete. But both, separatedness and completeness are not always easy to check.

THEOREM. If $\left(E_{n}\right)$ is an inductive sequence of normed spaces $E_{n}$ with closed unit balls $K_{n}$, such that, for all sequences $\left(\varepsilon_{m}\right)$ of positive numbers and all $n \in \mathrm{N}, \sum_{m=1}^{n} \varepsilon_{m} K_{m}$ is closed in $E_{n+1}$, then $E=$ ind $_{n \rightarrow} E_{n}$ is regular, in particular Hausdorff.

Proof. The method is more or less standard. Let $A \subset E$ be $\tau$-bounded and not $\tau_{n}$-bounded for all $n$.

(a) Assume that there are $\varepsilon_{m}>0$ and $x_{m} \in A$ with

$$
\frac{1}{m} x_{m} \notin \sum_{i=1}^{n} \varepsilon_{i} K_{i}=: U_{n}, \quad m=1,2, \ldots, n .
$$

Then, because $U_{n-1} \subset U_{n},(*)$ holds for all $m, n \in N$ :

$$
\frac{1}{m} x_{m} \notin U:=\bigcup_{n=1}^{\infty} U_{n}, \quad m=1,2, \ldots
$$

But $U$ is a $\tau$-neighbourhood of zero and $\left((1 / m) x_{m}\right) \tau$-converges to zero $(A$ being $\tau$-bounded)-a contradiction is established.

(b) Thus $\left(\varepsilon_{m}\right)$ and $\left(x_{m}\right)$ with (*) have to be constructed: $A \not \subset K_{1}$, so there is an $x_{1} \notin K_{1}, \varepsilon_{1}:=1$. Proceeding by induction, assume that $\varepsilon_{m}>0$ and $x_{m} \in A$ with

$$
\frac{1}{m} x_{m} \notin \sum_{i=1}^{n} \varepsilon_{i} K_{i}=: U_{n}, \quad m<n,
$$

are given. By the assumption of the theorem $U_{n}$ is closed in $E_{n+1}$; thus there is an $\varepsilon_{n+1}$ with

$$
(1 / m) x_{m} \notin U_{n}+\varepsilon_{n+1} K_{n+1}=: U_{n+1}, \quad m<n
$$

(the $x_{m}$ need not be elements of $E_{n+1}$ ). Since $U_{n+1}$ is bounded in $E_{n+1}$, the set $A$ is not contained in $(n+1) U_{n+1}$ and this implies the existence of an $x_{n+1} \in A$ with

$$
\frac{1}{n+1} x_{n+1} \notin U_{n+1}=\sum_{i=1}^{n+1} \varepsilon_{i} K_{i}
$$

4. It is clear that the assumption of the theorem must only be satisfied for, say, rapidly decreasing sequences $\left(\varepsilon_{n}\right)$. But the summation of closed sets is always problematic which is illustrated by a theorem of V. Klee [10, \$24.4] which states that a quasi-complete locally convex space is semi-reflexive if 
and only if the sum of each two convex, bounded, closed sets is closed (see $\$ 8$ for an example).

COROllaRy 1. Let $\left(E_{n}\right)$ be an inductive sequence of normed spaces, $F$ a semireflexive locally convex space and $T$ an injective, continuous operator $E=\operatorname{ind}_{n \rightarrow} E_{n} \rightarrow F$ such that $T K_{n}$ is closed for all $n$. Then $E=\operatorname{ind}_{n \rightarrow} E_{n}$ is regular.

Proof. All $T K_{n}$ are $\sigma\left(F, F^{\prime}\right)$-compact and so is

$$
\sum_{m=1}^{n} \varepsilon_{m} T K_{m}=T\left(\sum_{m=1}^{n} \varepsilon_{m} K_{m}\right)
$$

in particular closed: $T$ being injective and continuous yields that the theorem applies.

Take for example Köthe echelon-spaces: The $E_{n}$ are of the form ( $\omega$ denotes the Fréchet-Schwartz space of all real or complex sequences, $a_{k}>0$ )

$$
l^{1}\left(a_{k}\right):=\left\{\left(\xi_{k}\right) \in \omega\left|\left\|\left(\xi_{k}\right)\right\|:=\sum_{k=1}^{\infty}\right| \xi_{k} \mid a_{k}<\infty\right\} .
$$

The unit ball of $l^{1}\left(a_{k}\right)$ is closed and bounded in $\omega$, so Corollary 1 gives:

If $0<a_{k}^{n+1}<a_{k}^{n}$ for all $k, n \in \mathrm{N}$ then the space ind $l_{n \rightarrow} l^{1}\left(a_{k}^{n}\right)$ is regular.

This is a result of E. Dubinsky [2, Proposition 2].

Injective, continuous operators $T: G \rightarrow F$ between Banach spaces which map the closed unit ball onto a closed set are studied by H. P. Lotz, N. T. Peck, and H. Porta [11] and are called by them semi-embeddings.

5. Another use of the fact that the sum of compact sets is compact yields

COROLlaRY 2. The inductive limit of a sequence of dual Banach spaces such that the inclusion mappings are dual mappings is regular.

Proof. With $E_{n}=G_{n}^{\prime}$, the unit ball (in the dual norm) in $E_{n}$ is $\sigma\left(E_{n}, G_{n}\right)$ compact and the inclusion map is $\sigma\left(E_{n}, G_{n}\right)-\sigma\left(E_{n+1}, G_{n+1}\right)$ continuous.

The unit ball in an equivalent norm of a dual Banach space $G^{\prime}$ need not be $\sigma\left(G^{\prime}, G\right)$-compact: It is easily checked that

$$
e=(1,1, \ldots) \notin K:=\left\{\left(\xi_{n}\right) \in l^{\infty} \mid \max \left(\left\|\left(\xi_{n}\right)\right\|_{\infty}, 2 \lim \sup \left|\xi_{n}\right|\right)<1\right\}
$$

but $e$ is in the $\sigma\left(l^{\infty}, l^{1}\right)$-closure of $K$ (this norm is from an example in [11]). The existence of such a norm in the dual characterizes nonreflexive Banach spaces [8, p. 155].

Corollary 2 can also be proven by the observation that $\operatorname{proj}_{\leftarrow n} G_{n}^{\prime}$ is quasibarrelled (strongly bounded sets in the dual are equicontinuous).

6. The familiar fact that inductive limits of sequences of locally convex spaces with (weakly) compact linking mappings are regular [4], [9] is a consequence of Corollary 2, using, for example, the following known lemma 
[7, pp. 104-105]; [11]. (For a bounded, absolutely convex subset $A$ of a locally convex space, $\llbracket A \rrbracket$ denotes the linear hull of $A$ equipped with the Minkowski norm $m_{A}$.)

LEMMA. Let $K$ be a absolutely convex, weakly compact subset of a locally convex space $E$, then there is a Banach-space $B$ such that $\llbracket K \rrbracket=B^{\prime}$ isometrically and $\llbracket K \rrbracket=B^{\prime} \hookrightarrow E$ is $\sigma\left(B^{\prime}, B\right)-\sigma\left(E, E^{\prime}\right)$ continuous.

(Take $B=E^{\prime} /$ ker $m_{K^{0}}$ with the quotient norm of $m_{K^{0}}$ and notice that the unit ball of $B^{\prime}$ in $E^{\prime \prime}$ is $K^{00}=K$.)

7. E. Dubinsky [3] gave a sufficient condition for an inductive limit to be Hausdorff which turned out being very useful. The present method gives

COROLlaRY 3. If $\left(E_{n}\right)$ is an inductive sequence of normed spaces such that the bidual mappings $E_{n}^{\prime \prime} \rightarrow E_{n+1}^{\prime \prime}$ are injective, then $E=\operatorname{ind}_{n \rightarrow} E_{n}$ is Hausdorff and $\beta$-regular.

PRoof. $\hat{E}:=\operatorname{ind}_{n \rightarrow} E_{n}^{\prime \prime}$ is regular by Corollary 2, in particular Hausdorff; since the map $E=$ ind $E_{n} \hookrightarrow \hat{E}$ is continuous and injective this implies, that $E$ is also Hausdorff. A bounded set $A \subset E$ is bounded in $\hat{E}$ and thus situated and bounded in some $E_{n}^{\prime \prime}$ : If $A$ is in $E_{m}$ then it is bounded in $E_{k}, k=$ $\max (m, n)$, since $E_{k} \subset E_{k}^{\prime \prime}$ as a subspace.

In particular all inductive limits (definitions similar to that of $l^{1}\left(a_{k}\right)$ ) $\operatorname{ind}_{n \rightarrow} c_{0}\left(a_{k}^{n}\right)$ are Hausdorff and $\beta$-regular because the bidual of $c_{0}\left(a_{k}\right) \hookrightarrow$ $c_{0}\left(b_{k}\right)$ is $l^{\infty}\left(a_{k}\right) \hookrightarrow l^{\infty}\left(b_{k}\right)$, E. Dubinsky (unpublished) constructed such a sequence of $c_{0}$ 's which is not regular.

8. The difficulty applying the theorem comes from V. Klee's characterization of nonreflexive spaces. That the closed sets with a nonclosed sum are not all pathological is shown with the following example. It shows also drastically why the theorem does not help in the case of strict embeddings (i.e., $E_{n}$ being a subspace of $E_{n+1}$ )-but this is not severe because there is a very general result available (e.g. [6, p. 127]).

There is a (necessarily nonreflexive) Banach-space with a closed hyperplane $H, K$ the unit ball, such that $K+K \cap H$ is not closed.

Proof. Consider in $c_{0}$, with the sup-norm \|\|$_{\infty}$, the closed hyperplane

$$
H:=\left\{\left(\xi_{n}\right)_{n=1}^{\infty} \in c_{0} \mid \sum_{n=1}^{\infty} \xi_{n} \frac{1}{2^{n}}=0\right\} .
$$

(a) If $\left(\xi_{n}\right) \in K \cap H$ then $\xi_{1}<1$, since otherwise

$$
\frac{1}{2}=\sum_{n=2}^{\infty}\left(-\xi_{n}\right) \frac{1}{2^{n}}
$$

which is impossible because of $\left|\xi_{n}\right|<1$ and $\xi_{n} \rightarrow 0$.

(b) This implies $2 e_{1}:=(2,0,0, \ldots) \notin K+K \cap H$.

(c) If $0<\varepsilon<1$ then there is an $x_{\varepsilon}=(1-\varepsilon, \ldots) \in K \cap H$ : Take 


$$
d=\sum_{n=2}^{N} \frac{1}{2^{n}}>\frac{1-\varepsilon}{2}
$$

and

$$
x_{\varepsilon}=\left(1-\varepsilon, \frac{\varepsilon-1}{2 d}, \ldots, \frac{\varepsilon-1}{2 d}, 0, \ldots\right),
$$

the zeros starting in component $N+1$.

(d) Since $(2-\varepsilon) e_{1}-x_{\varepsilon} \in K$ it follows that

$$
2 e_{1}=\lim _{\varepsilon \rightarrow 0}\left[(2-\varepsilon) e_{1}-x_{\varepsilon}+x_{\varepsilon}\right] \in \overline{K+K \cap H} .
$$

This, together with (b) establishes the desired example.

Does the existence of such a hyperplane characterize nonreflexive Banachspaces? Using the theorem of R. C. James on the supremum of linear functionals, more or less the same proof as above shows that this is true up to a slight modification of the norm.

\section{REFERENCES}

1. M. DeWilde, Sur un type particulier de limite incuctive, Bull. Soc. Roy. Sci. Liège 35 (1966), 545-551.

2. E. Dubinsky, Echelon spaces of order $\infty$, Proc. Amer. Math. Soc. 16 (1965), 1178-1183.

3. __ Projective and inductive limits of Banach spaces, Studia Math. 42 (1972), 259-263.

4. K. Floret, Lokalkonvexe Sequenzen mit kompakten Abbildungen, J. Reine Angew. Math. 247 (1971), 155-195.

5. , Folgenretraktive Sequenzen lokalkonvexer Raume, J. Reine Angew. Math. 259 (1973), 65-85.

6. K. Floret and J. Wloka, Einführung in die Theorie der lokalkonoexen Raume, Lecture Notes in Math., vol. 56, Springer-Verlag, Berlin and New York, 1968.

7. A. Grothendieck, Produits tensoriels topologiques et espaces mucléaires, Mem. Amer. Math. Soc. No. 16 (1955).

8. R. B. Holmes, Geometric functional analysis and its applications, Graduate Texts in Mathematics, no. 24, Springer-Verlag, New York, 1975.

9. H. Komatsu, Projective and injective limits of weakly compact sequences of locally convex spaces, J. Math. Soc. Japan 19 (1967), 366-383.

10. G. Köthe, Topological vector spaces. I, Die Grundlehren der math. Wissenschaften, Band 159, Springer-Verlag, New York, 1966.

11. H. P. Lotz, N. T. Peck and H. Porta, Semi-embeddings of Banach-spaces (to appear).

12. B. M. Makarov, Pathological properties of inductive limits of Banach-spaces, Uspehi Mat. Nauk 18 (1963), 171-178 (Russian).

Department of Mathematics, State Universtty of New York, Buffalo, New York 14214

Mathematisches Seminar der Universität, D-23 Kiel, Bundesrepublix Deutschland (Current address) 